\title{
Two breakthrough gene-targeted treatments for spinal muscular atrophy: challenges remain
}

\author{
Charlotte J. Sumner ${ }^{1,2}$ and Thomas 0. Crawford ${ }^{1,3}$ \\ 'Department of Neurology, ${ }^{2}$ Department of Neuroscience, and ${ }^{3}$ Department of Pediatrics, Johns Hopkins University School of Medicine, Baltimore, Maryland, USA.
}

\begin{abstract}
The motor neuron disease spinal muscular atrophy (SMA) is caused by recessive, loss-of-function mutations of the survival motor neuron 1 gene (SMN1). Alone, such mutations are embryonically lethal, but SMA patients retain a paralog gene, SMN2, that undergoes alternative pre-mRNA splicing, producing low levels of SMN protein. By mechanisms that are not well understood, reduced expression of the ubiquitously expressed SMN protein causes an early-onset motor neuron disease that often results in infantile or childhood mortality. Recently, striking clinical improvements have resulted from two novel treatment strategies to increase SMN protein by (a) modulating the splicing of existing SMN2 pre-mRNAs using antisense oligonucleotides, and (b) transducing motor neurons with self-complementary adeno-associated virus 9 (scAAV9) expressing exogenous SMN1 cDNA. We review the recently published clinical trial results and discuss the differing administration, tissue targeting, and potential toxicities of these two therapies. We also focus on the challenges that remain, emphasizing the many clinical and biologic questions that remain open. Answers to these questions will enable further optimization of these remarkable SMA treatments as well as provide insights that may well be useful in application of these therapeutic platforms to other diseases.
\end{abstract}

In the century following its original description in 1891 (1), the degenerative neuromuscular disorder spinal muscular atrophy (SMA) was considered one of the most hopeless of maladies. A shift in that fortune began in 1995 , when a unique digenic molecular mechanism of pathogenesis was delineated (2). All patients harbor inactivating (often large deletion) mutations of the survival motor neuron 1 gene (SMN1), but copy number of an adjacent, partially functional paralog gene, SMN2, scales inversely with phenotypic severity $(3,4)$. Thus both genes were defined as attractive targets for therapeutic manipulation, and a fortunate series of therapy-enabling basic and clinical science advances followed. On the basic science side, there was a rapidly evolving science of RNA splicing biology, synthetic antisense oligonucleotide (ASO) development, refinement of gene transfer vectors, and a number of informative disease-manifesting mouse models. On the clinical side, rigorous natural history studies, the development of meaningful, reliable, and sensitive disease outcome measures, and experience in early clinical trials yielded important insights that enabled efficient trial design. SMA is common enough to support investment, and patient advocacy groups were well organized to support clinical trials. The result of this cascade of good fortune is the demonstration of two new powerful therapies, recently published in the New England Journal of Medicine (5-7).

Conflict of interest: CJS has been a consultant to Avexis, Ionis Pharmaceuticals, Biogen, SMA Foundation, PTC Therapeutics, and Roche. CJS received a research grant from lonis Pharmaceuticals in $2016(\$ 75,000)$.CJS is a coholder of 2 pending patent applications (BIOL0274USA and BIOL0293WO) with lonis Pharmaceuticals on antisense oligonucleotides targeting SMN-AS1. TOC has been a consultant to lonis Pharmaceuticals, Biogen, and Avexis. He is/has been a site principal investigator for the EMBRACE and NURTURE Biogen clinical trials and the current STRIVE and STRONG Avexis trials.

Reference information: J Clin Invest. 2018;128(8):3219-3227.

https://doi.org/10.1172/JCl121658.

\section{Spinal muscular atrophy}

The two SMN genes, telomeric SMN1 and centromeric SMN2, reside in each portion of a large inverted duplication of the chromosome $5 \mathrm{q} 13$ region that arose during primate evolution (ref. 8 and Figure 1A). Both are constitutively transcribed. The functionally relevant difference between SMN1 and SMN2 is a single base pair $(\mathrm{C} \rightarrow \mathrm{T})$ in exon 7 that does not change the protein sequence but does alter SMN2 pre-mRNA processing by largely directing splicing from the exon 6 donor to the exon 8 acceptor, thus excluding exon 7 (9-12). This shortened mRNA encodes a truncated, more rapidly degraded SMN protein (Figure 1B). Importantly, a small portion of the SMN2 transcript retains exon 7, which then produces some normal SMN protein. This amount of normal SMN is sufficient to rescue most cells from the degeneration that accompanies complete absence of protein. Why motor neurons are selectively vulnerable to diminished abundance of SMN protein is unknown. SMN has multiple putative functions, but the best established is in regulating the assembly of spliceosomal small nuclear ribonuclear proteins (snRNPs) (13-15), key constituents of the spliceosome that are essential to eukaryotic pre-mRNA processing.

The clinical manifestations of SMA arise primarily from motor neuron degeneration with associated skeletal muscle denervation, weakness, and muscle atrophy. It manifests across a wide range of severity, extending from severe, infantile-onset to mild, adult-onset (Figure 2A). The pattern of weakness, concentrated in proximal and truncal muscles but also affecting diaphragm and distal muscles of the limbs, is consistent across the spectrum of severity (16). Those with the most severe form of SMA, known as type I, constitute about $60 \%$ of affected individuals and tend to retain only two copies of the SMN2 gene. These infants, after a few weeks or months of apparent good health, develop progressive skeletal muscle weakness that, absent intervention, leads to fatal respirato- 
A

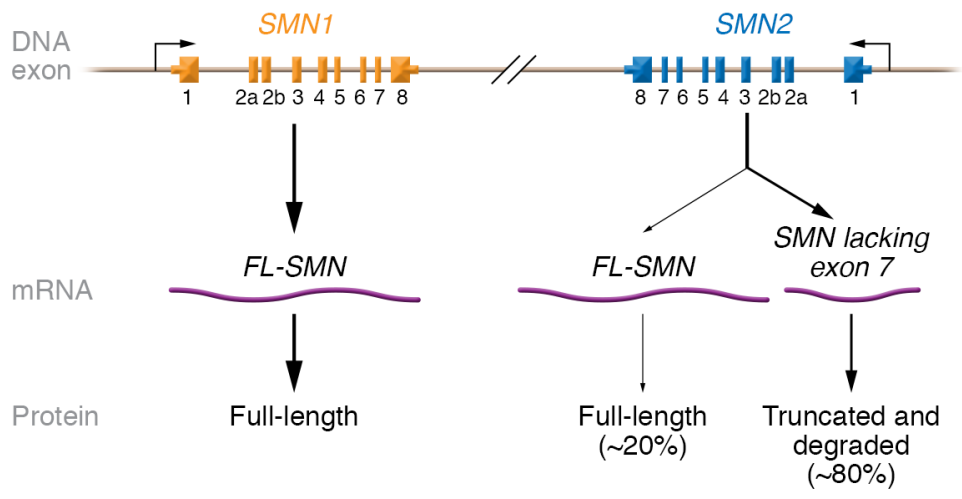

B

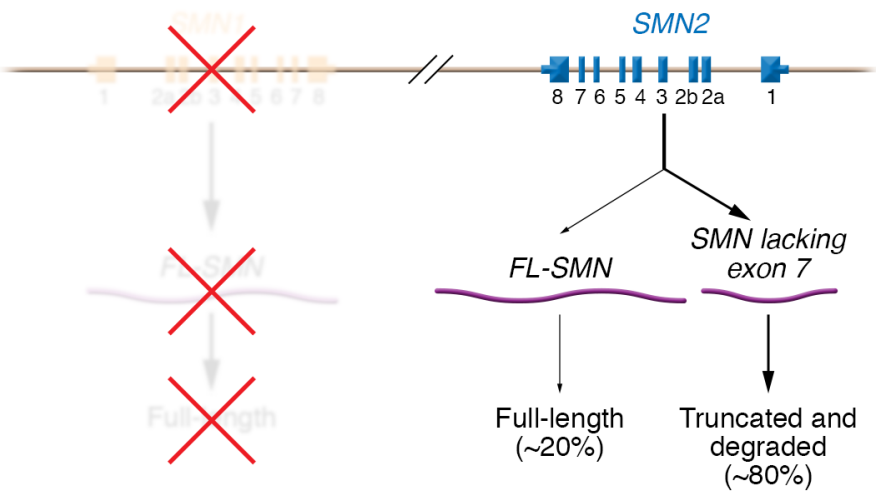

C

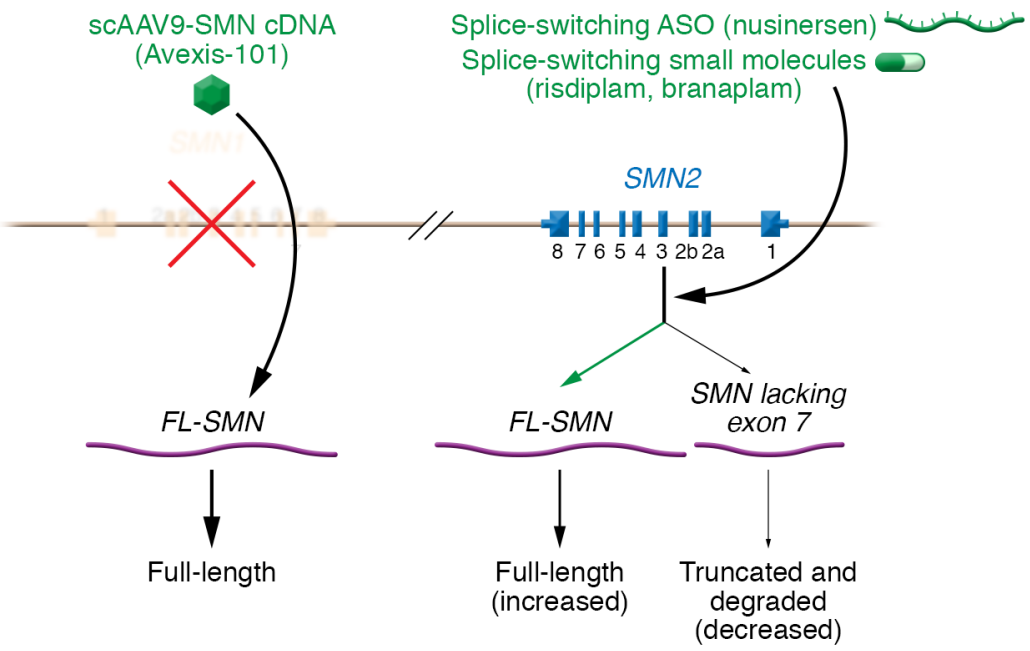

Figure 1. The SMN1 and SMN2 genes that cause SMA can each be targeted by different therapeutic strategies. (A) SMN1 and SMN2 gene expression in a healthy individual. (B) Schematic of SMA genetics. (C) Therapeutic mechanism of scAAV9-SMN1 CDNA, the splice-switching ASO, and the small molecules risdiplam (RG7916) and branaplam (LMIO70) currently in clinical trials (see Table 1).

may be subtle, such that prospective studies of affected children have been unable to measure decline over a 1-year period $(17,19)$. Longer duration and retrospective experience of older children and adults, however, reliably demonstrate that the apparent "plateau" phase is characterized by slow loss of motor abilities and muscle power (authors' personal clinical practice observations). Prior to the new therapies described in this article, clinical care of SMA patients was exclusively supportive, largely addressing the downstream respiratory, orthopedic, and nutritional consequences of weakness that contribute to morbidity $(20,21)$. In practice, the level of supportive care for infants with type I SMA has ranged widely from exclusively palliative to intensive life-extending respiratory and nutritional support. Critical to the design of infantile treatment trials, however, was demonstration that aggressive supportive care, while extending duration of life, failed to improve upon the natural history of persistent, profound muscle weakness associated with failure to achieve any major motor milestone $(17,22)$.

\section{The therapies}

\section{Nusinersen/Spinraza (ASO)}

The first therapy targets the partially functioning SMN2 paralog by enhancing exon 7 inclusion during splicing of SMN2 premRNAs (Table 1 and Figure 1C). ASOs are single-stranded nucleic acids designed to hybridize to their target RNA sequence by Watson-Crick base pairing. Depending on their structure, ASOs can be designed to target select mRNAs for RNase-H-mediated degradation; or, as in SMA, they can bind pre-mRNAs and act as a steric hindrance to protein binding or RNA secondary structure (23). A key advance in understanding the basic mechanisms that regulate SMN2 exon 7 splicing was the identification of a negative regulatory element in intron 7, intronic splicing silencer N1 (ISS-N1), which binds the splicing repressor hnRNP A1 $(24,25)$. ASOs that hybridize to ISS-N1

ry insufficiency around the first birthday $(17,18)$. Those inheriting three or more copies of SMN2 tend to milder weakness that appears at later ages. At the mildest end of the disease spectrum are those who will not manifest weakness until young adulthood or even later. SMA is also unusual in that, unlike many neurodegenerative disorders, the rate of emerging clinical weakness is greatest at the outset of the course, followed thereafter by more gradual worsening (Figure 2A). Progression of weakness in the later, chronic phase increased exon 7 inclusion and full-length SMN protein expression in SMA patient-derived fibroblasts (24), and an optimized ASO developed by Ionis Pharmaceuticals markedly attenuated disease in rodent models of SMA (26-28).

In general, endonucleases rapidly degrade short sequences of single-stranded nucleic acid in vivo, limiting their potential as therapeutic agents. Chemical modifications of the oligonucleotide backbone and sugar rings have led to molecules with 
A

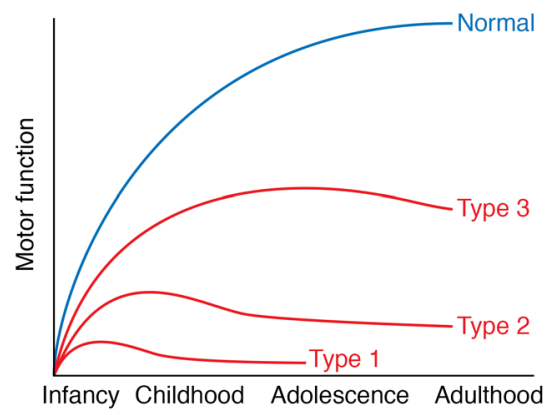

B

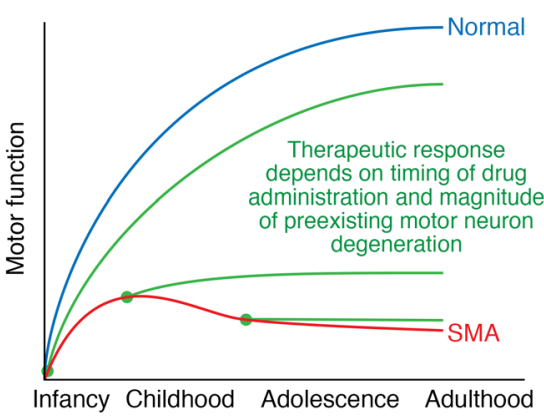

Figure 2. The clinical course of SMA and alterations with treatment. (A) A schematic depiction of the usual clinical course of SMA types I, II, and III. The general trend of functional loss is greatest at the outset of disease and diminishes thereafter. In some cases, the earliest course manifests as departure from the path of normal development, with some transient gains before loss of ability is seen. (B) Green lines indicate variable hypothetical therapeutic responses, which depend on timing of drug administration (green circles) and the magnitude of preexisting motor neuron degeneration. properties that are key to pharmaceutical application, including resistance to endonuclease digestion, suppression of TLR-mediated innate immune activation, and an increased affinity to targeted RNA (23). Nusinersen has a phosphothioate backbone with a uniform 2'-O-methoxyethyl ribose modification that has shown a good safety profile in humans and rodents (29). Naked ASOs are taken up by many cell types into lysosomal or endosomal compartments and then into the nucleus through incompletely understood mechanisms. As ASOs do not transverse the blood-brain barrier, targeting the CNS in humans requires intermittent intrathecal injection. Initial concern about limited CNS distribution following intrathecal infusion was countered by empiric data showing widespread and effective spinal cord and motor neuron penetration in rodents and nonhuman primates (30). The long persistence of nusinersen within CNS tissue of animal models and the first-in-humans intrathecal dosing experience of an ASO during a phase I trial in familial ALS (31) led to the human treatment trial protocol of four loading doses over 2 months followed by maintenance dosing at 4- or 6-month intervals. Early-phase safety and pharmacokinetic studies in SMA subjects progressed quickly (32, 33), enabling registration-level clinical trials.

\section{Avexis-101 (scAAV9-SMN)}

By delivering an exogenous SMN1 cDNA, the second therapeutic program follows a different strategy to restore SMN protein expression in vulnerable cells (Table 1 and Figure 1B). The path here was built on the foundation of basic work on viral vectors.
Experience with a range of viral vectors (34), including retrovirus, lentivirus, adenovirus, and herpes virus, led to interest in the nonpathogenic adeno-associated virus (AAV) family because of a number of promising qualities, including their potential to transduce neurons (35). AAVs are small, nonenveloped, single-stranded DNA viruses that require coinfection of a "helper" adenovirus for replication. Recombinant AAVs principally establish themselves as a persistently expressing episome with little incorporation into the host genome. Because they do not replicate with cell division, recombinant AAVs are diluted over time in mitotically active tissues, but virus may be very long-lived in terminally differentiated cells such as motor neurons - potentially allowing one-time dosing. In one study, AAV2-mediated transgene expression was evident in human brain 10 years after administration (36). The minimal or absent host genome integration reduces concern about oncogenesis, and diminished inflammatory and immune responses to AAVs improve their tolerability in comparison with other viral vectors. Although they have a limited packaging capacity, replacement of the native AAV genome with a desired cDNA and promoter offers an ideal means for gene transfer therapy. To overcome the delay in transgene expression caused by the requirement for second-strand synthesis, double-stranded, self-complementary recombinant AAV vectors (scAAVs) have been designed that speed protein synthesis following transduction.

Different AAV serotypes have preferential tissue tropism, and the breakthrough that allowed the use of AAV gene transfer for SMA was the finding that the AAV9 serotype effectively targets motor neurons when administered intravascularly, particularly in

Table 1. Differing routes and frequency of administration of SMN-inducing therapies

\begin{tabular}{|c|c|c|c|c|}
\hline Drug (manufacturer) & $\begin{array}{l}\text { Nusinersen } \\
\text { (Biogen/lonis) }\end{array}$ & $\begin{array}{l}\text { scAAV9-SMN } \\
\text { (Avexis) }\end{array}$ & $\begin{array}{c}\text { Risdiplam } \\
\text { (Roche/PTC/SMA Foundation) }\end{array}$ & $\begin{array}{l}\text { Branaplam } \\
\text { (Novartis) }\end{array}$ \\
\hline Mechanism & $\begin{array}{l}\text { SMN2 splice-switching } \\
\text { ASO }\end{array}$ & $\begin{array}{l}\text { Viral-mediated SMN1 } \\
\text { gene replacement }\end{array}$ & $\begin{array}{l}\text { SMN2 splice-switching } \\
\text { small molecule }\end{array}$ & $\begin{array}{l}\text { SMN2 splice-switching } \\
\text { small molecule }\end{array}$ \\
\hline Frequency & $\begin{array}{l}4 \text { loading doses over } 2 \text { months, } \\
\text { then every } 4 \text { months }\end{array}$ & Once & Daily & Daily \\
\hline Current SMA target patient population & FDA-approved for all & Type I SMA & All & Type I SMA \\
\hline
\end{tabular}




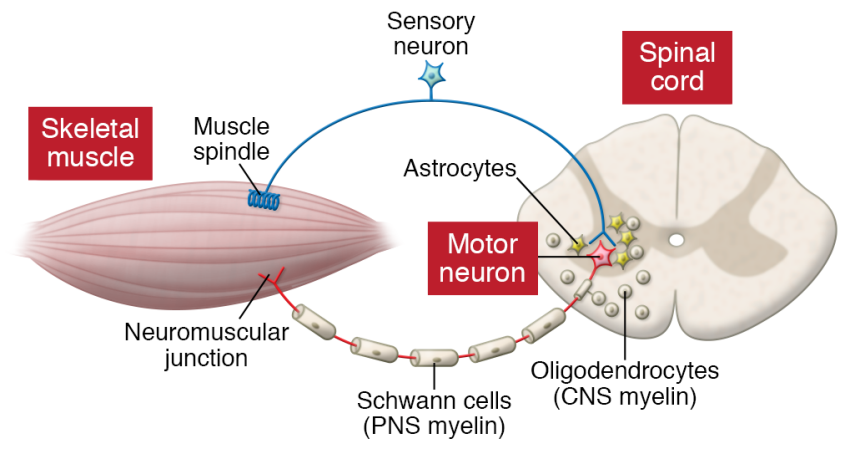

Figure 3. Schematic of cell types directly interacting with motor neurons. The motor unit is composed of multiple cell types that may be affected directly or secondarily by SMN deficiency.

young mice, cats, and primates (37-39). High doses of systemically injected virus are required to achieve robust CNS transduction, but the mechanism used by AAV9 to cross the blood-brain barrier is not known. Preclinical efficacy studies of scAAV9 expressing SMN1 cDNA showed marked improvements of disease phenotypes in SMA mouse models (40-42). The dose and temporal dependency for efficacy observed in these preclinical studies guided decisions regarding the dosing of human patients. With this preclinical development, and successful resolution of the substantial regulatory and manufacturing challenges, the single-site phase I study of single-dose intravenous dosing with Avexis-101 (AVXS-101) in SMA infants at Nationwide Children's Hospital at Ohio State University was initiated.

\section{The trials}

\section{What is common to both trials}

The two trials targeted infants predicted to manifest the severe, type I form of SMA (refs. 5, 6, and Table 2). Selection of this group of patients, in whom extension of ventilator-free survival could be evaluated quickly, was considered the most likely path to demonstrate a meaningful clinical outcome. Targeting this group for initial trials also addressed the greatest clinical need. Notably, infants diagnosed at a young age as a consequence of manifest clinical weakness never gain expected developmental motor skills. Both trials were happily surprised not only by improvements in survival, but also by unexpected and significant improvements in motor function.

\section{What is unique?}

The Biogen nusinersen ASO trial was designed as a phase III registration-level sham-controlled blinded study. One hundred twenty-two infants were randomly enrolled at a 1:2 ratio to receive sham intrathecal injections or intrathecal administration of nusinersen in a loading series at $0,2,4$, and 8 weeks, followed by maintenance doses every 4 months thereafter. The trial was halted at a predetermined interval of 13 months when the unblinded monitor identified a significant improvement in motor outcomes in the treated cohort. At study completion, 39\% of infants in the nusinersen group met the defined endpoint of death or permanent assisted ventilation compared with $68 \%$ of the sham group, resulting in a $47 \%$ reduced risk of death or permanent ventilation. The most dramatic finding was that $51 \%$ of nusinersen-treated infants (37 of 73 ) compared with $0 \%$ of sham-treated infants ( 0 of 37 ) had measurably improved motor ability. In the nusinersen group, 22\% achieved full head control, $8 \%$ were able to sit independently, and $1 \%$ were able to stand.

In contrast, the Avexis AAV9-SMN gene transfer trial was a pilot, open-label trial of 15 SMA infants intended to study dosage and safety of single-dose intravenous scAAV9-SMN and to provide an early look at efficacy as assessed by duration of survival and motor function. After 3 patients tolerated a low dose of viral genomes $\left(6.7 \times 10^{13} \mathrm{vg} / \mathrm{kg}\right.$ body weight), approval was granted to dose the next 12 subjects at a higher dose $\left(2 \times 10^{14} \mathrm{vg} / \mathrm{kg}\right.$ body weight). Notably, this is the highest dose of any viral vector that has been administered to a human subject. At the time of publication, all infants (by then older than 20 months of age) were alive, and 11 of 12 infants in the higher-dose group had significant gains in motor function, 11 sitting independently, 9 rolling over, and 2 walking independently. A multicenter, single-arm trial comparing scAAV9-SMN-treated infants with infantile SMA against historical controls is now being conducted (NCT03306277, ClinicalTrials.gov), along with an additional pilot, phase I study of preschool-aged children to whom scAAV9-SMN is administered intrathecally (NCTO3381729) (43).

\section{Which is better?}

Direct head-to-head comparison of the two infant trials is not possible. There are important differences in the age of treated subjects - those in the AAV9-SMN trial were younger (3.4 months in the high-dose group) than those in the nusinersen trial (5.4 months). Outcome measures also differed. Importantly, the scAAV9-SMN gene transfer study is unblinded. It is clear that neither therapy delivered in this fashion is a cure. Subjects in each group manifested a range of outcomes, from little to no improvement, to substantial gains in motor function, and all will likely manifest some motor deficits lifelong. For the moment, the focus should rightly be on the remarkable benefits of both therapies for a disease that previously followed an unwavering degenerative path.

\section{Will these therapies benefit other groups of SMA patients?}

After only a 3-month review, the US Food and Drug Administration approved nusinersen in December 2016 as a treatment for individuals of all ages with SMA. This indication was probably based on the logic that SMA disease manifestations are driven by SMN protein deficiency in all patient types and that this mechanism can be therapeutically targeted regardless of age. Partial support for this argument comes from similar salutary motor findings in a companion double-blind, sham-controlled trial of 126 children between 2 and 12 years of age (with recruitment biased to younger subjects within this range) (Table 2 and ref. 7). Like the infantile trial, this trial was halted at the interim analysis time point of 15 months. Improved motor function in this cohort of more mildly affected children was seen in $57 \%$ of those receiving nusinersen as opposed to $26 \%$ of the sham control group.

The most striking results with nusinersen were observed in an ongoing trial of 25 presymptomatic SMA infants first treated before 6 weeks of age ( 15 harboring two SMN2 copies and 10 harboring three SMN2 copies) (Table 2). Interim data have been 
Table 2. Notable recent or ongoing clinical trials involving SMN-inducing drugs

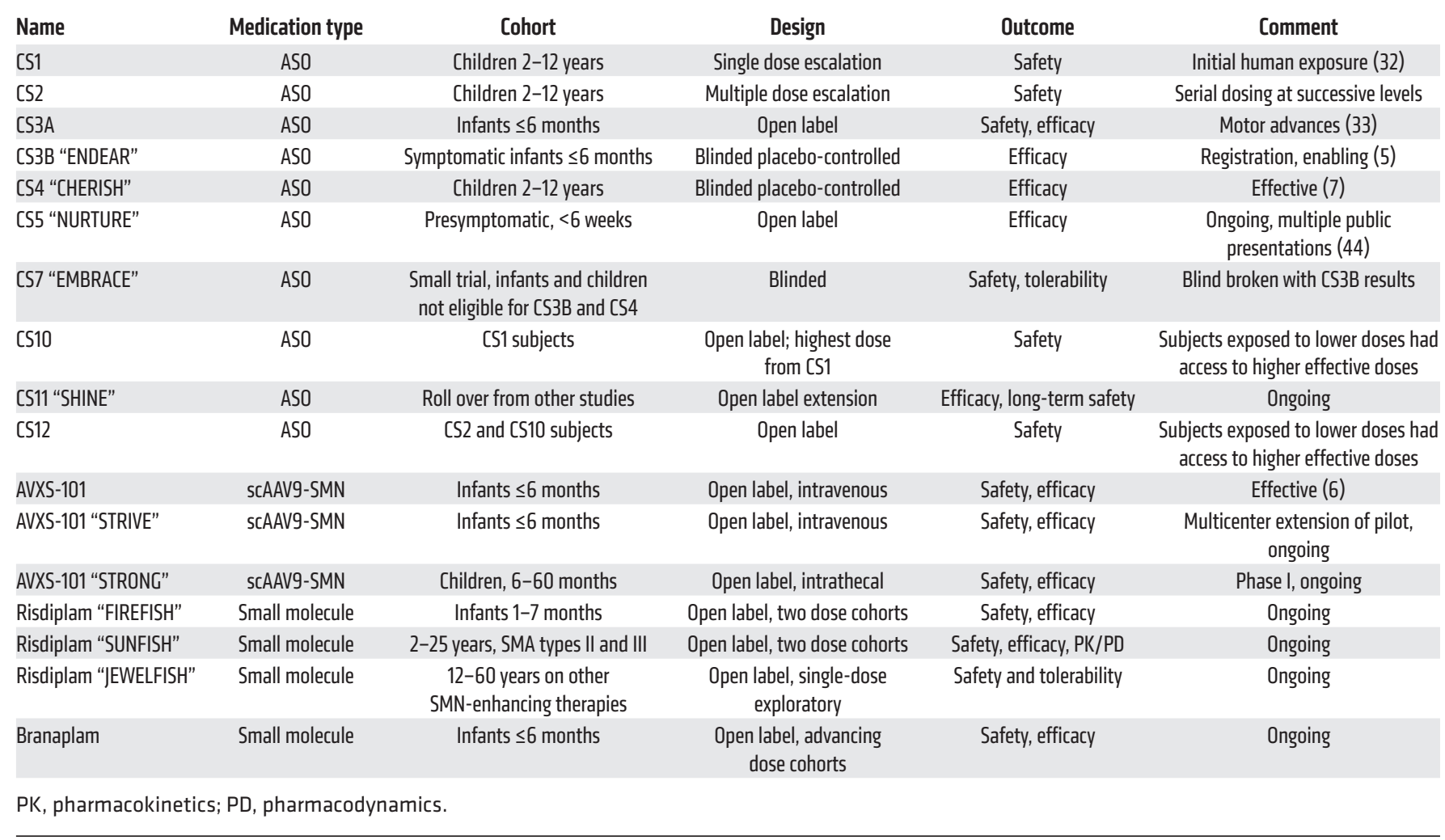

publicly presented but remain unpublished (44). After approximately 1.5 years of treatment, $100 \%$ of children sit independently, $53 \%$ stand unaided, and 50\% walk independently. At least a few of these children, particularly those with three copies of SMN2, appear to be tracking along a fully normal path of development.

Taken together, the clinical trial data to date suggest that two related factors affect outcome. The first is age at time of treatment, as within and between all of the trials, as well as in preclinical studies $(40,45,46)$, a trend to better outcome with earlier treatment is clear. The magnitude of treatment effect also doubtless relates to the number of motor neurons yet to succumb. Early treatment before onset of motor neuron loss or development of clinical symptoms is ideal. A notable development in parallel with these therapeutic advances has been development and validation of a newborn genetic screening protocol for SMA (47). The American Advisory Committee on Heritable Disorders in Newborns and Children recently recommended nationwide newborn screening for SMA in the United States; further action at the national and state level is still required before implementation.

An important caveat, however, applies to how these trials in children will apply to older individuals with SMA. The trial-demonstrated speed and magnitude of treatment benefit in infants parallel the gains in muscle power and motor function that are normally expected with development (Figure 2B). Treatment-associated improvements of motor function may thus represent an unveiling of normal developmental gains that are made possible by suppression of early-childhood disease-associated progressive motor neuron loss. This distinction is important in considering potential outcomes of treatment of those beyond the age of expected developmental gains. While older individuals may not manifest short-term improvements in motor function, they may well greatly benefit by stabilization of function against the natural history of very slow worsening.

\section{Potential toxicities}

The rapid development of these programs precludes assessment of long-term complications of either therapy. The first human exposure to nusinersen was in 2011, and to scAAV9-SMN in 2014 (Table 2). Nusinersen's preclinical and clinical experience was remarkable for the absence of complications. Renal toxicity has been reported for ASOs with different backbone chemistries and routes of administration, but only small percentages of nusinersen-exposed subjects showed mild elevations of urinary protein. Similarly, severe thrombocytopenia has been observed with some systemically delivered ASOs, but only $11 \%$ in one of the nusinersen trials showed a mild reduction of platelets (none below 50,000 cells $/ \mu \mathrm{l}$ ). Routine assessment of complete blood count and urinary protein is recommended with each dose. Of greater impact, however, is the substantial burden associated with the requirement for repeated intrathecal administration. While lumbar punctures (LPs) are often well tolerated, complications of post-LP headache and local pain are common in older children and adults. Many individuals with chronic SMA have associated spinal deformity or prior spinal fusion that necessitates fluoroscopic posterior or lateral transforaminal approaches to the intrathecal space, with an associated increased risk of complications. There is great interest in other long-term strategies for easing the burden of repeated administration. 
AAV is not known to be associated with any human disease, yet detection of antibodies to various AAV serotype capsid proteins, including AAV9, is frequent (48), indicating that exposure and probable asymptomatic infection are common. The presence of antibodies is currently an exclusion to treatment. Another issue is potential inflammatory reactions that may occur at the time of dosing. Pretreatment with oral prednisone was initiated after the first trial subject developed asymptomatic increased liver transaminase levels; at trial's end, four of the treated cohort had transaminase elevations without other indication of compromised liver function. A recent study of an intravenously administered, high-dose AAV9 variant (AAVhu68) expressing human SMN in three nonhuman primates and piglets observed examples of life-threatening fulminant liver toxicity and dorsal root ganglion neuron degeneration (49). Although this is a different viral serotype and such toxicities have not been seen in patients, this study does indicate that further work is needed to understand why such responses can occur in certain circumstances and raises the potential for idiosyncratic toxicity when larger cohorts are exposed to the virus. Another concern is potential insertional genotoxicity leading to oncogenesis, particularly in the liver, where multiple copies per cell of the AAV9 encapsulated genome capsid might accumulate, increasing the risk. Whether or not AAV encapsulated vector incorporation presents a genotoxic threat remains controversial (50-52), but long-term anticipated risks should be considered in the context of potential benefit to subjects at high risk for disease complication.

\section{Other SMN-inducing treatments on the horizon}

Remarkably, in addition to nusinersen and AAV9-SMN, two other SMN-inducing treatments are currently in clinical trials in SMA patients (Figure 1, Tables 1 and 2). A collaborative group including the SMA Foundation, PTC Therapeutics, and Roche, and an independent effort by Novartis, sought to identify orally bioavailable, brain-penetrant small molecules to enhance exon 7 inclusion into the SMN2 transcripts. Both efforts successfully identified small molecules $(53,54)$ that have demonstrated remarkable efficacy in SMA mouse models, similar to that achieved with ASO and AAV9 approaches. The challenge of limiting off-target effects of these small molecules has somewhat slowed both drug development programs. The small-molecule trials are mainly being carried out outside of the United States, as the potential path to registration for a second drug is difficult when there is already an approved therapy.

\section{Real challenges remain}

An unfortunate casualty arising from dramatic success is the misperception that SMA has been "solved." The opposite is true at every level of basic and clinical research, as success inevitably breeds new questions.

\section{Optimizing drug dosing and cell targeting}

Are there greater gains to be had? The motor function improvements in both trials of symptomatic infants lag well behind normal development, and SMN-enhancement programs may not yet be optimal. While early treatment maximizes effect, many of these infants lag behind a normal development pathway, and all may yet experience a ceiling of potential motor achievement.
Issues related to drug pharmacokinetics. The specifics of ASO or AAV9 distribution and durability of effect in humans are unknown and difficult to study, as the targeted spinal cord cannot be sampled during life. Limited data obtained at autopsy in three SMA type I infants dosed with nusinersen in the phase II trial (33) confirmed broad immunohistochemical distribution of nusinersen after intrathecal dosing. Not unexpectedly, drug concentrations were highest in the caudal regions and diminished at rostral levels. The relationship between tissue levels and efficacy is not yet known, but an important observation is that truncal and respiratory muscles respond less well than do limb muscles in many children treated with nusinersen. Possible factors affecting response include flow dynamics of cerebrospinal fluid, variation in the efficacy of drug uptake, and the real possibility that SMN-deficient motor neurons are limited in their ability to take up ASO or AAV9 at some stage in disease pathogenesis. Comparison of regional responses between intrathecally delivered ASO and intravenously administered AAV9 may be informative.

A fortunate constraint to the study of human spinal cord distribution of AAV9-SMN is that none of the children dosed have died. Optimization of dose will depend on greater clinical experience. Animal models suggest that enhanced spinal cord (30) transduction is possible with intrathecal administration of the vector, which has the advantage of greatly decreasing the required dose and thus hepatic exposure and cost of manufacture, but adds the concerns about potential regional deficiencies of CNS distribution in humans that confound the ASO experience. Antibodies to AAV9 present a special concern for AAV9 therapy that has yet to be worked out. AAV9 antibodies can also be transiently present in infants due to maternal transfer. One infant in the Avexis trial was excluded because of above-threshold titers of AAV9 antibody (6). Induction of antibodies after AAV9 dosing would likely preclude repeated gene therapy treatment. The threshold titer at which antibodies affect transduction is unknown.

Issues related to drug pharmacodynamics. Both therapies work by increasing the level of SMN protein, though by very different mechanisms. The magnitude and speed of SMN induction are doubtless different for the two therapies, as is the threshold of each needed for maximum efficacy. It is likely that scAAV9-SMN induces SMN protein more rapidly than ASOs. The magnitude of SMN induction by splice-switching ASOs is limited by the amount of available $S M N 2$ pre-mRNA template, whereas virally delivered $S M N 1$ cDNA driven by the chicken $\beta$-actin promoter does not have this constraint. Pharmacodynamic studies, like pharmacokinetic studies, are constrained by the targeted spinal cord tissue's inaccessibility for biochemical testing. The only opportunity arises from autopsy tissues, which may bias toward poor responders to either therapy. In the three nusinersen-treated infant autopsies discussed above (33), there was an increase in the ratio of full-length to truncated SMN2 transcripts in spinal cord as well as increased SMN immunohistochemical staining intensity in motor neurons, indicating an ability to modify SMN expression. While encouraging, this early evidence does not provide insight into ASO levels needed for SMN induction, or SMN levels needed to forestall disease progression. Unfortunately, efforts to reliably detect SMN protein levels in cerebrospinal fluid have not yet been successful, thus precluding human treatment monitoring studies. 
There is an urgent need for biomarkers to assess early stages of SMN repletion within motor neurons or other affected tissues.

Issues related to cell types targeted. The principal clinical manifestations of SMA likely result from diminished SMN protein within motor neurons themselves. Notably, however, experimental evidence indicates that decreases of SMN protein levels in any tissue can lead to pathology. In severe SMA mouse models, abnormalities of multiple cell and tissue types have been described (reviewed in refs. 55, 56). The threshold at which this occurs is likely affected by tissue type, species, age, and other factors. Several concerns arise from these two findings. First is the possibility that individuals with SMA who have had CNS SMN rescue by ASO or AAV9 approaches will eventually manifest signs and symptoms of peripheral SMN deficiency. There are multiple reports of nonCNS organ pathology in humans with SMA, though a direct relationship to SMN deficiency as opposed to other causes, including downstream complications of profound weakness, is not established. A second concern is whether or not other SMN-deficient tissues may play a contributing role to motor neuron degeneration. Impairment of cells and tissues that directly interact with motor neurons within and outside the CNS could contribute to pathology (Figure 3). While CNS-delivered ASO treatment of a severe SMA mouse model improved outcome, additional systemic ASO provided an additional boost (28). Muscle, in particular, is a candidate for this supporting role in motor neuron stability, as profound myofiber hypotrophy and overall sarcopenia are early pathologies in SMA, and SMN deficiency undermines numerous direct and indirect circulating factors ("myokines") that provide potential support (57). If these extraneuronal contributors are relevant, the small-molecule approaches to SMN induction would target nonCNS tissues more efficiently than scAAV9 or ASOs.

\section{Other concerns}

Will the SMA phenotype be altered by regional differences in response to treatment? While it is attractive to think of SMN-enhancing treatments as simply improving the severity of weakness along established patterns, treatment initiated after emergence of clinical weakness, or even onset of nascent pathology, may well change the appearance of the SMA phenotype. This is because the timing of degeneration differs between motor neuron pools. Across the range of SMA severity and age, denervation generally affects proximal skeletal muscles first and most severely, especially those of the chest and abdominal wall. The diaphragm is relatively spared, producing a unique "abdominal" pattern of breathing and special difficulty with airway clearance. Treatment after bulbar and chest wall muscle weakness is well established but, at a time when appendicular motor neuron pools can still be rescued, may increase the relative burden placed on respiratory and pharyngeal muscles, affecting airway clearance or swallowing functions.

What is the effect of SMN enhancement over time? Both the longterm biologic threshold needed for SMN and the durability of SMN induction associated with the two therapies are unknown. The continuation of slow decline in patients of all degrees in adulthood suggests that SMN deficiency retains pathologic importance in patients at all ages, though at least one study in SMA mouse models has suggested that sufficiently early SMN repletion can be associated with safe withdrawal later in life (58). Matching these unknowns is uncertainty about the durability of SMN enhancement within cells after treatment by either therapy. The present recommended schedule calling for three yearly maintenance doses of nusinersen is based on limited nonhuman primate data (30). Treatment with AAV9 is promoted as a single lifelong durable treatment given persistence of the AAV9 episome in animal models, but there is little information to establish the extent to which this experience is applicable to humans.

\section{Dosing of patients with chronic disease and a need for combinatorial approaches}

While we do not yet know the full therapeutic impact of presymptomatic treatment in the forthcoming era of newborn screening, hints from early treatment of patients with two copies of SMN2 suggest it is not a cure. Certainly treatment after onset of weakness constrains achievable responses in a progressive manner (Figure 2B). The achievable level of a treatment's "clinically meaningful benefit" necessarily scales with age and preexisting disease. In those who have advanced to the more chronic clinical phase of expected slow worsening, maintenance of motor function over a many-year timeframe or modest improvements in motor function rather than gains of major motor milestones should be the expectation. It is important to note that many with chronic SMA have devised exceptional means to function despite profound weakness, and hence the meaningfulness of small improvements, or simply of stabilization, is greatest in those individuals who are weakest. Further clinical studies will be needed to carefully evaluate such effects in the patients not evaluated in these initial clinical trials.

In addition, a priority for future translational research should be to characterize potentially important underlying cellular events that mediate clinical responses in different patient groups. While the large benefit arising from treatment of infants may be dominated by reversal of impaired development, more modest improvements in older patients may be mediated by distinct remodeling events such as improvement of the fidelity of transmission across existing neuromuscular junctions, increasing of motor unit size by increased sprouting of motor neuron axons, or recovery of functional excitability of motor neurons that are in a dysfunctional pre-degenerative state (59). Insight into these questions will enable therapeutic development efforts to optimize or possibly combine the current approaches but may also uncover new therapeutic targets that can be combined with SMN induction to optimize therapeutic efficacy. There are ongoing efforts to evaluate the efficacy of tropomyosin activators and myostatin inhibitors to improve muscle function and/or bulk in combination with SMN induction (60).

\section{Other challenges}

Both therapies involve novel biologic mechanisms that were developed at high cost and with substantial risk. Financing in capital markets is thus accompanied by the expectation of higher return on investment, a return that must be proportioned over a relatively small group. Commercial Spinraza is a leading example of the new era of "personalized medicine" in which costs are exceptionally high and present a challenge to traditional insurance systems. The dramatic success of these two therapies, con- 
centrated in sympathetic children who easily claim public attention, make this a highly visible example of this trend. As it is, many patients who would benefit from nusinersen are not yet able to access it. How society and governments respond to this in the context of limited resources but proliferating health care costs will emerge with time.

\section{Relevance of similar approaches to other diseases}

SMA was exceptionally well positioned to take advantage of advances in basic and clinical medicine that led to these therapies. Refinement of ASO and scAAV9 viral transfer therapies for treatment of SMA thus affords many insights that will be of value to other therapeutic development programs. The single target (SMN), unidimensional disease manifestation (skeletal muscle weakness), and relative frequency of the disorder that facilitates full recruitment to clinical trials all provided a simplicity that enabled successful development. Success of the SMA programs will almost certainly be relevant to design of programs targeting other disorders in which outcome measures are more complex. ASO and scAAV9 replacement strategies have been proposed for a wide range of neurologic disorders $(23,35,61,62)$, and the lessons learned from SMA thus have wide-ranging implications.

\section{Acknowledgments}

CJS is supported by the National Institute of Neurological Disorders and Stroke (NINDS) R01NS096770, Cure SMA, SMA Research Team, and the SMA Foundation.

Address correspondence to: Charlotte J. Sumner, Departments of Neurology and Neuroscience, Johns Hopkins University School of Medicine, 855 North Wolfe Street, Rangos 234, Baltimore, Maryland 21205, USA. Phone: 410.502.6085; Email: csumner1@jhmi.edu.
1. Werdnig G. Zwei frühinfantile hereditäre fälle von progressive muskelatrophie unter dem bilde der dystrophie, aber auf neurotischer grundlage. Arch fur Psychiatr Nervenkrankh. 1891;22:437-481.

2. Lefebvre $S$, et al. Identification and characterization of a spinal muscular atrophy-determining gene. Cell. 1995;80(1):155-165.

3. Lefebvre S, et al. Correlation between severity and SMN protein level in spinal muscular atrophy. Nat Genet. 1997;16(3):265-269.

4. Parsons DW, McAndrew PE, Iannaccone ST, Mendell JR, Burghes AH, Prior TW. Intragenic telSMN mutations: frequency, distribution, evidence of a founder effect, and modification of the spinal muscular atrophy phenotype by cenSMN copy number. Am J Hum Genet. 1998;63(6):1712-1723.

5 . Finkel RS, et al. Nusinersen versus sham control in infantile-onset spinal muscular atrophy. $N$ Engl JMed. 2017;377(18):1723-1732.

6. Mendell JR, et al. Single-dose gene-replacement therapy for spinal muscular atrophy. $\mathrm{N} \mathrm{EnglJ}$ Med. 2017;377(18):1713-1722.

7. Mercuri E, et al. Nusinersen versus sham control in later-onset spinal muscular atrophy. $N$ Engl J Med. 2018;378(7):625-635.

8. Rochette CF, Gilbert N, Simard LR. SMN gene duplication and the emergence of the SMN2 gene occurred in distinct hominids: SMN2 is unique to Homo sapiens. Hum Genet. 2001;108(3):255-266.

9. Lorson CL, Hahnen E, Androphy EJ, Wirth B. A single nucleotide in the SMN gene regulates splicing and is responsible for spinal muscular atrophy. Proc Natl Acad Sci U S A. 1999;96(11):6307-6311.

10. Monani UR, et al. A single nucleotide difference that alters splicing patterns distinguishes the SMA gene SMN1 from the copy gene SMN2. Hum Mol Genet. 1999;8(7):1177-1183.

11. Cartegni L, Krainer AR. Disruption of an SF2/ ASF-dependent exonic splicing enhancer in SMN2 causes spinal muscular atrophy in the absence of SMN1. Nat Genet. 2002;30(4):377-384.

12. Kashima T, Manley JL. A negative element in SMN2 exon 7 inhibits splicing in spinal muscular atrophy. Nat Genet. 2003;34(4):460-463.

13. Liu Q, Dreyfuss G. A novel nuclear structure containing the survival of motor neurons protein.
EMBO J. 1996;15(14):3555-3565

14. Liu Q, Fischer U, Wang F, Dreyfuss G. The spinal muscular atrophy disease gene product, SMN, and its associated protein SIP1 are in a complex with spliceosomal snRNP proteins. Cell. 1997;90(6):1013-1021.

15. Fischer U, Liu Q, Dreyfuss G. The SMN-SIP1 complex has an essential role in spliceosomal snRNP biogenesis. Cell. 1997;90(6):1023-1029.

16. Crawford TO, Pardo CA. The neurobiology of childhood spinal muscular atrophy. Neurobiol Dis. 1996;3(2):97-110.

17. Finkel RS, et al. Observational study of spinal muscular atrophy type I and implications for clinical trials. Neurology. 2014;83(9):810-817.

18. Kolb SJ, et al. Natural history of infantileonset spinal muscular atrophy. Ann Neurol. 2017;82(6):883-891.

19. Kaufmann P, et al. Prospective cohort study of spinal muscular atrophy types 2 and 3. Neurology. 2012;79(18):1889-1897.

20. Mercuri E, et al. Diagnosis and management of spinal muscular atrophy. Part 1: Recommendations for diagnosis, rehabilitation, orthopedic and nutritional care. Neuromuscul Disord. 2018;28(2):103-115.

21. Finkel RS, et al. Diagnosis and management of spinal muscular atrophy. Part 2: Pulmonary and acute care; medications, supplements and immunizations; other organ systems; and ethics. Neuromuscul Disord. 2018;28(3):197-207.

22. De Sanctis R, et al. Developmental milestones in type I spinal muscular atrophy. Neuromuscul Disord. 2016;26(11):754-759.

23. Schoch KM, Miller TM. Antisense oligonucleotides: translation from mouse models to human neurodegenerative diseases. Neuron. 2017;94(6):1056-1070.

24. Singh NK, Singh NN, Androphy EJ, Singh RN. Splicing of a critical exon of human Survival Motor Neuron is regulated by a unique silencer element located in the last intron. Mol Cell Biol. 2006;26(4):1333-1346.

25. Singh NN, Howell MD, Androphy EJ, Singh RN. How the discovery of ISS-N1 led to the first medical therapy for spinal muscular atrophy. Gene Ther. 2017;24(9):520-526.
26. Hua Y, Vickers TA, Okunola HL, Bennett CF, Krainer AR. Antisense masking of an hnRNP A1/A2 intronic splicing silencer corrects SMN2 splicing in transgenic mice. Am J Hum Genet. 2008;82(4):834-848

27. Passini MA, et al. Antisense oligonucleotides delivered to the mouse CNS ameliorate symptoms of severe spinal muscular atrophy. Sci Transl Med. 2011;3(72):72ra18.

28. Hua Y, et al. Peripheral SMN restoration is essential for long-term rescue of a severe spinal muscular atrophy mouse model. Nature. 2011;478(7367):123-126.

29. Rigo F, Hua Y, Krainer AR, Bennett CF. Antisense-based therapy for the treatment of spinal muscular atrophy. J Cell Biol. 2012;199(1):21-25.

30. Rigo F, et al. Pharmacology of a central nervous system delivered 2'-O-methoxyethyl-modified survival of motor neuron splicing oligonucleotide in mice and nonhuman primates. J Pharmacol Exp Ther. 2014;350(1):46-55.

31. Miller TM, et al. An antisense oligonucleotide against SOD1 delivered intrathecally for patients with SOD1 familial amyotrophic lateral sclerosis: a phase 1 , randomised, first-in-man study. Lancet Neurol. 2013;12(5):435-442.

32. Chiriboga CA, et al. Results from a phase 1 study of nusinersen (ISIS-SMN(Rx)) in children with spinal muscular atrophy. Neurology. 2016;86(10):890-897.

33. Finkel RS, et al. Treatment of infantile-onset spinal muscular atrophy with nusinersen: a phase 2, open-label, dose-escalation study. Lancet. 2016;388(10063):3017-3026.

34. Serguera C, Bemelmans AP. Gene therapy of the central nervous system: general considerations on viral vectors for gene transfer into the brain. Rev Neurol (Paris). 2014;170(12):727-738.

35. Saraiva J, Nobre RJ, Pereira de Almeida L. Gene therapy for the CNS using AAVs: the impact of systemic delivery by AAV9. J Control Release. 2016;241:94-109.

36. Leone P, et al. Long-term follow-up after gene therapy for canavan disease. Sci Transl Med. 2012;4(165):165ra163.

37. Foust KD, Nurre E, Montgomery CL, Hernandez A, Chan CM, Kaspar BK. Intravascular AAV9 
preferentially targets neonatal neurons and adult astrocytes. Nat Biotechnol. 2009;27(1):59-65.

38. Duque $S$, et al. Intravenous administration of self-complementary AAV9 enables transgene delivery to adult motor neurons. Mol Ther. 2009;17(7):1187-1196.

39. Bevan AK, et al. Systemic gene delivery in large species for targeting spinal cord, brain, and peripheral tissues for pediatric disorders. Mol Ther. 2011;19(11):1971-1980.

40. Foust KD, et al. Rescue of the spinal muscular atrophy phenotype in a mouse model by early postnatal delivery of SMN. Nat Biotechnol. 2010;28(3):271-274.

41. Valori CF, et al. Systemic delivery of scAAV9 expressing SMN prolongs survival in a model of spinal muscular atrophy. Sci Transl Med. 2010;2(35):35ra42.

42. Dominguez E, et al. Intravenous scAAV9 delivery of a codon-optimized SMN1 sequence rescues SMA mice. Hum Mol Genet. 2011;20(4):681-693.

43. Meyer K, et al. Improving single injection CSF delivery of AAV9-mediated gene therapy for SMA: a dose-response study in mice and nonhuman primates. Mol Ther. 2015;23(3):477-487.

44. New data presented at MDA Clinical Conference show benefit in motor function for infants, teens and young adults treated with SPINRAZA (nusinersen) [press release]. Cambridge, Massachusetts, USA: Business Wire; March 12, 2018. http://investors.biogen.com/news-releases/ news-release-details/new-data-presentedmda-clinical-conference-show-benefit-motor.
Accessed June 19, 2018.

45. Narver HL, et al. Sustained improvement of spinal muscular atrophy mice treated with trichostatin $\mathrm{A}$ plus nutrition. Ann Neurol. 2008;64(4):465-470.

46. Lutz CM, et al. Postsymptomatic restoration of SMN rescues the disease phenotype in a mouse model of severe spinal muscular atrophy. J Clin Invest. 2011;121(8):3029-3041.

47. Phan HC, Taylor JL, Hannon H, Howell R. Newborn screening for spinal muscular atrophy: anticipating an imminent need. Semin Perinatol. 2015;39(3):217-229.

48. Boutin S, et al. Prevalence of serum IgG and neutralizing factors against adeno-associated virus (AAV) types 1, 2, 5, 6, 8, and 9 in the healthy population: implications for gene therapy using AAV vectors. Hum Gene Ther. 2010;21(6):704-712.

49. Hinderer $\mathrm{C}$, et al. Severe toxicity in nonhuman primates and piglets following high-dose intravenous administration of an adeno-associated virus vector expressing human SMN. Hum Gene Ther. 2018;29(3):285-298.

50. Chandler RJ, Sands MS, Venditti CP. Recombinant adeno-associated viral integration and genotoxicity: insights from animal models. Hum Gene Ther. 2017;28(4):314-322.

51. $\mathrm{Li} \mathrm{H}$, et al. Assessing the potential for AAV vector genotoxicity in a murine model. Blood. 2011;117(12):3311-3319.

52. Byrne BJ. Safety first: perspective on patient-centered development of AAV gene therapy products. Mol Ther. 2018;26(3):669-671.

53. Naryshkin NA, et al. Motor neuron disease.
SMN2 splicing modifiers improve motor function and longevity in mice with spinal muscular atrophy. Science. 2014;345(6197):688-693.

54. Palacino J, et al. SMN2 splice modulators enhance U1-pre-mRNA association and rescue SMA mice. Nat Chem Biol. 2015;11(7):511-517.

55. Hamilton G, Gillingwater TH. Spinal muscular atrophy: going beyond the motor neuron. Trends Mol Med. 2013;19(1):40-50.

56. Nash LA, Burns JK, Chardon JW, Kothary R, Parks RJ. Spinal muscular atrophy: more than a disease of motor neurons? Curr Mol Med. 2016;16(9):779-792.

57. Giudice J, Taylor JM. Muscle as a paracrine and endocrine organ. Curr Opin Pharmacol. 2017;34:49-55.

58. Kariya S, et al. Requirement of enhanced survival motoneuron protein imposed during neuromuscular junction maturation. J Clin Invest. 2014;124(2):785-800.

59. Crawford TO. Concerns about the design of clinical trials for spinal muscular atrophy. Neuromuscul Disord. 2004;14(8-9):456-460.

60. Groen EJN, Talbot K, Gillingwater TH. Advances in therapy for spinal muscular atrophy: promises and challenges. Nat Rev Neurol.2018;14(4):214-224.

61. Havens MA, Hastings ML. Splice-switching antisense oligonucleotides as therapeutic drugs. Nucleic Acids Res. 2016;44(14):6549-6563.

62. Choudhury SR, Hudry E, Maguire CA, Sena-Esteves M, Breakefield XO, Grandi P. Viral vectors for therapy of neurologic diseases. Neuropharmacology. 2017;120:63-80. 